\title{
Frecuencia del síndrome de diesgaste profesional en un grupo de especialistas en Cirugía Plástica y estudiantes del posgrado en Colombia
}

\section{Frequency of hurnout syndrome in a group of specialists in Plastic Surgery and postgraduate students in Colombia}

John VARGAS RUEDA*, Jorge GAVIRIA PINZÓN**, Ginna Paola SAAVEDRA MARTÍNEZ***, Ricardo GALÁN SUÁREZ****

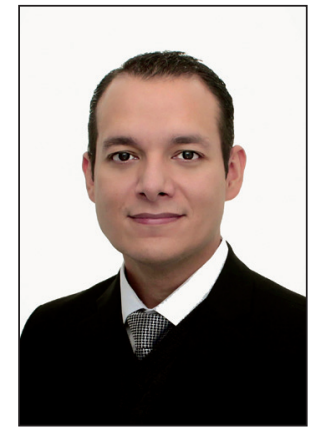

Vargas Rueda J.
Resumen

Introducción y objetivo. El síndrome burnout o síndrome de desgaste profesional, fue descrito por Freudenberger, psiquiatra psicoanalista norteamericano en 1974, y empleado públicamente por primera vez por Cristina Maslach en 1976 para referirse a una situación cada vez más frecuente entre trabajadores de los servicios humanos, que por la naturaleza de su trabajo, debían mantener un contacto directo y continuo con la gente, desgastándose profesionalmente tras meses o años de dedicación. En los 80, Maslach creó un instrumento para su valoración, el Maslach Burnout Inventory (MBI), con 3 versiones, una de los cuales tiene como objetivo las personas que trabajan en el sector de servicios de la salud, que valora 3 dimensiones: agotamiento emocional, despersonalización y realización personal en el trabajo, que sigue vigente y es considerado el instrumento más utilizado y acertado para el diagnóstico de la enfermedad.

El objetivo de nuestro estudio es determinar la frecuencia y factores asociados del síndrome de desgaste profesional en médicos especialistas y estudiantes de posgrado en Cirugía Plástica, Estética y Reconstructiva en Colombia.

Material y método. Estudio de corte transversal durante el XX Curso Internacional de Cirugía Plástica Estética de la Sociedad Colombiana de Cirugía Plástica, Estética y Reconstructiva (SCCP) de septiembre 2018 en Barranquilla. Solicitamos consentimiento informado y utilizamos el cuestionario Maslach Burnout Inventory y un cuestionario sociodemográfico para evaluar los factores asociados con el síndrome. Definimos el burnout o síndrome de desgaste profesional como la asociación de alto agotamiento emocional, despersonalización y bajo rendimiento profesional. Realizamos el análisis multivariado después del ajuste del modelo logístico binario con la identificación de los factores de riesgo y el calculo de (OR). De los 623 médicos registrados en el curso, 132 participaron en el estudio.

Resultados. Dos de los 98 especialistas evaluados presentaban criterios de síndrome de desgaste profesional. Dos especialistas fueron excluidos por presentar depresión mayor previamente diagnosticada. Ninguno de los residentes evaluados cumplió con todos los criterios para la entidad. Sin embargo, identificamos diferentes porcentajes en los encuestados que podrían encontrarse en riesgo, así como factores evaluables en las personas con mejores puntajes que podrían jugar un papel fundamental en su prevención.

Conclusiones. El síndrome de desgaste profesional o burnout requiere mayor atención en cuestión de salud pública. Estudios como el nuestro permiten exponer factores psicosociales que podrían ser evaluados para identificar no solo factores de riesgo, sino aquellos que puedan ser protectores para el desarrollo de esta entidad, y así crear estrategias de prevención y reducir la incidencia de esta patología.

\begin{tabular}{|lr|}
\hline Palabras clave & $\begin{array}{l}\text { Síndrome de desgaste profesional, } \\
\text { Agotamiento profesional, Sobrecarga } \\
\text { emocional, Fatiga en el trabajo, Cirugía }\end{array}$ \\
$\begin{array}{l}\text { Plástica. } \\
\text { Nivel de evidencia científica }\end{array}$ & 5 c Diagnóstico \\
Recibido [esta versión] & 28 julio / 2020 \\
Aceptado & 13 febrero / 2021
\end{tabular}

Background and objective. The burnout was originally described by Freudenberger, an American psychoanalyst psychiatrist in 1974, and this term was used for the first time by Cristina Maslach in 1976 to refer to an increasingly frequent situation among workers of human services, who by the nature of their work, had to maintain direct and continuous contact with people, wasting out professionally after months or years of dedication. In the 1980s, Maslach created an instrument for its assessment, the Maslach Burnout Inventory (MBI), with 3 versions, one aimed at people who work in the human services sector (Human Services Survey). It values 3 dimensions: emotional exhaustion, depersonalization and personal accomplishment at work, is still in use and is considered the most accurate instrument for diagnosing the disease.

The objective of this study was to determine the frequency and associated factors of burnout syndrome in a group of plastic surgeons and postgraduate students in Aesthetic and Reconstructive Plastic Surgery in Colombia.

Methods. Cross-sectional study carried out during the XX International Course of Aesthetic Plastic Surgery of the Colombian Society of Aesthetic and Reconstructive Plastic Surgery (SCCP) carried out in September 2018 in Barranquilla. Informed consent was requested and the Maslach Burnout Inventory questionnaire and a sociodemographic questionnaire were used to evaluate the factors associated with the syndrome. Burnout syndrome was defined as the association of high emotional exhaustion, depersonalization and low personal accomplishment. The multivariate analysis was performed after fitting the binary logistic model with the identification of risk factors and the calculation of the (OR). Of the 623 physicians registered in the course, 132 participated in the study.

Results. Two of the 98 specialists evaluated meet the criteria for burnout syndrome. Two specialists were excluded for having previously diagnosed major depression. None of the residents evaluated met all the criteria for this entity. However, different percentages of respondents who could be at risk were identified, as well as different factors in people with the best scores that could play a fundamental role in their prevention.

Conclusions. The burnout syndrome requires more attention in terms of public health. Studies like ours allow to expose psychosocial factors to identify not only risk factors but those playing a protective role for the development of this entity, to create prevention strategies and to reduce the incidence of this pathology.

Keywords Burnout syndrome, Professional burnout, Emotional overload syndrome, Fatigue syndrome at work, Plastic surgery.

\section{Level of evidence Received [this version] Accepted} $5 c$ Diagnostic July 28 / 2020

Conflicto de intereses: Los autores declaran no tener ningún interés financiero relacionado con el contenido de este artículo. Financiación: No hubo fuentes externas de financiación para este trabajo.

* Cirujano Plástico, Universidad Militar Nueva Granada y Hospital Militar Central de Colombia, Bogotá, Colombia.

** Médico Residente de Cirugía Plástica, Universidad Militar Nueva Granada y Hospital Militar Central de Colombia, Bogotá, Colombia.

*** Epidemióloga Clínica, Unidad de Investigación Científica Hospital Militar Central de Colombia, Bogotá, Colombia.

*** Cirujano Plástico, Subespecialista en Cirugía de la Mano, Especialista en Pedagogía y Docencia, Profesor Titular de la Facultad de Medicina de la Universidad Militar Nueva Granada y Profesor Emérito del Hospital Militar Central de Colombia, Bogotá, Colombia. 


\section{Introducción}

El síndrome burnout o síndrome de desgaste profesional hace referencia a una situación cada vez más frecuente entre trabajadores, principalmente en los profesionales de la salud y docentes. Fue descrito originalmente por Freudenberger, un psiquiatra psicoanalista norteamericano en 1974. El término fue empleado públicamente por primera vez por Cristina Maslach en 1976 para referirse a una situación cada vez más frecuente entre trabajadores de los servicios humanos, quienes por la naturaleza de su trabajo debían mantener un contacto directo y continuo con la gente, desgastándose profesionalmente después de meses o años de dedicación. ${ }^{(1)}$ En la década de los 80 Maslach creó un instrumento para su valoración, el Maslach Burnout Inventory (MBI), con 3 diferentes versiones, una de los cuales tiene como objetivo las personas que trabajan en el sector de servicios de la salud (Human Services Survey). Valora 3 dimensiones: el agotamiento emocional, la despersonalización y la realización personal en el trabajo. Sigue estando vigente y es el instrumento más utilizado y acertado para el diagnóstico de la enfermedad. ${ }^{(2-5)}$

Este síndrome aparece como una respuesta continua al estrés laboral y puede afectar a la salud física y mental del individuo así como a sus relaciones sociales y a su desempeño laboral, lo cual impacta negativamente en la prestación de servicios y a la relación médico-paciente. Cuando los profesionales de salud se ven afectados por el síndrome comienzan a sentirse irritables, aumentan sus ausencias laborales, bajan la calidad de su atencióny presentan alteraciones del sueño, entre otros síntomas que pueden llevarlos al consumo de sustancias, a la automedicación con psicofármacos o al consumo de drogas ilegales. Su manifestación y sus síntomas más habituales son:

- Psicosomáticos: fatiga crónica, trastornos del sueño, úlceras y desordenes gástricos y tensión muscular.

- De conducta: ausentismo laboral y adicciones (tabaco, alcohol, drogas).

- Emocionales: irritabilidad, incapacidad de concentración y distanciamiento afectivo.

- Laborales: menor capacidad laboral, acciones hostiles y conflictos. ${ }^{(4)}$

En general, de acuerdo con Maslach, se acepta que las dimensiones que contribuyen a delimitar dicho síndrome son:

- Cansancio o agotamiento emocional: constituye la primera fase del proceso caracterizado por una progresiva pérdida de energía y una desproporción creciente entre el trabajo realizado y el cansancio experimentado.
- En esta etapa las personas se vuelven más irritables, aparece la queja constante por la cantidad de trabajo realizado perdiendo la capacidad de disfrutar de las tareas. Desde una mirada externa, se empieza a percibir como una persona permanentemente insatisfecha, quejosa e irritable.

- Despersonalización: es una forma de responder a los sentimientos de impotencia, indefensión y desesperanza personal. En lugar de expresar estos sentimientos y resolver los motivos que los originan, las personas que padecen este síndrome muestran una fachada hiperactiva que incrementa su sensación de agotamiento. En esta fase se alternan la depresión y la hostilidad hacia el medio.

- Abandono de la realización personal: consiste en la retirada progresiva de todas las actividades no laborales vinculadas a aquellas situaciones que generaron el estrés crónico. En esta etapa hay pérdida de ideales $y$, principalmente, una creciente ausencia de actividades familiares, sociales y recreativas, creando una especie de autorreclusión. ${ }^{(6-9)}$

Hay múltiples factores individuales y ambientales que conducen a situaciones de mayor desgaste profesional y a menor satisfacción en el trabajo, pero sin embargo, no existe acuerdo unánime con respecto a la etiología de este síndrome. Entre los factores individuales se han descrito algunos de riesgo para desarrollarlo como la edad, principalmente en personas jóvenes, de sexo femenino, solteras o sin pareja estable y personas con poco tiempo libre. También se han estudiado diversos rasgos de la personalidad, como el optimismo, la afectividad, la autoestima o determinados factores hereditarios. ${ }^{(10.11)}$

En cuanto a los profesionales de la salud, este síndrome cada vez cobra mayor importancia dado que se ha observado su impacto en la calidad de la atención a los pacientes, el ausentismo laboral y provoca pérdidas económicas al sector salud. Respecto a cómo influyen en ellos los factores ambientales, se ha observado que los profesionales de la salud son los que presentan mayores índices de desgaste profesional, insatisfacción, trastornos afectivos y estrés..$^{(12)}$

A partir de diversas teorías cabe destacar el síndrome de desgaste profesional como un fenómeno multicausal, complejo y poliédrico, en el que intervienen diferentes factores. En este sentido todo parece indicar que los valores personales y organizacionales tienen un peso específico en la explicación de esta patología. ${ }^{(13)}$

A la fecha y hasta donde hemos podido comprobar, no hay un estudio formal sobre la incidencia del Síndrome de desgaste profesional (burnout) en Cirugía Plástica con especialistas y residentes en nuestro entorno geográfico, motivo por el que nos propusimos desarrollar el siguiente estudio. 


\section{Material y método}

Realizamos un estudio descriptivo transversal durante el XX Curso Internacional de Cirugía Plástica Estética de la Sociedad Colombiana de Cirugía Plástica, Estética y Reconstructiva (SCCP) llevado a cabo en septiembre de 2018 en la ciudad de Barranquilla. La población objetivo fueron los especialistas y residentes de Cirugía Plástica, Estética y Reconstructiva que asistieron al curso. Recogimos los datos a través de cuestionarios autoadministrados manteniendo en todo momento la confidencialidad. Todos los participantes firmaron un formulario de consentimiento libre e informado.

Evaluamos el síndrome de desgaste profesional a través del Inventario de Burnout de Maslach (MBI) en su versión para personal de servicios sanitarios (Human Services Survey-HSS), una versión validada y adaptada al español en Colombia. ${ }^{(14)}$

El MBI-HSS consta de 22 preguntas subdivididas en las áreas de agotamiento emocional, despersonalización y realización personal. Los ítems son preguntas tipo Likert con 7 opciones, con la puntuación más baja (valor 0 ) nunca experimentado y la más alta cuando se experimenta diariamente (valor 6). Las primeras 9 preguntas corresponden al agotamiento emocional y se considera alto cuando la puntuación total es igual o mayor a 27 sobre un máximo de 54 puntos, utilizando los siguientes criterios de puntuación: bajo ( $\leq 18$ puntos), moderado (19 a 26 puntos) y alto ( $\geq 27$ puntos). La clasificación del dominio de despersonalización, con 5 preguntas, se realizó de acuerdo con la siguiente puntuación: bajo $(\leq 6$ puntos), moderado ( 7 a 12 puntos) y alto ( $\geq 13$ puntos). Para la realización personal, con 8 preguntas, la clasificación se realizó de acuerdo con el siguiente criterio: baja ( $\leq 33$ puntos), moderada ( 34 a 39 puntos) y alta ( $\geq 40$ puntos). El criterio utilizado en nuestro estudio para definir el síndrome de desgaste profesional fue la presencia de valores altos para los dominios de agotamiento emocional y despersonalización, combinados con una puntuación baja para el logro profesional.

También utilizamos un cuestionario sociodemográfico para evaluar los factores asociados con el síndrome de desgaste profesional. Consideramos la actividad física regular según la definición de la Organización Mundial de la Salud: más de 150 minutos semanales de actividad moderada (caminar rápido, bailar, participar activamente en juegos y deportes con niños y caminar con mascotas) o más de 75 minutos de actividad intensa (ciclismo, ejercicios aeróbicos, natación rápida, deportes y juegos competitivos).

Analizamos los datos utilizando el paquete estadístico STATA v.16 para evaluar el perfil personal y profesional, los hábitos, el nivel de actividad física y las condiciones de vida de los residentes que participaron en el estudio, calculamos los porcentajes y construimos las distribuciones de frecuencia de los factores evaluados. Usamos la prueba de chi-cuadrado para comparar proporciones.

Las variables secundarias para analizar los factores asociados con el síndrome de desgaste profesional, tanto en general como por componentes, fueron: género, edad, estado civil, ciudad de desempeño, número de hijos, horas de actividad física, turnos semanales, tabaquismo, consumo de alcohol, consumo de café, antecedente de consumo de sustancias psicoactivas (PSA), enfermedad incapacitante, diagnóstico de depresión mayor; para los especialistas consideramos además: tipo de práctica, horas de trabajo, tipo de contrato, antigüedad laboral, número de trabajos, vinculación a docencia, turnos nocturnos de trabajo, horas de sueño, horas de esparcimiento, actividades de esparcimiento y personas a cargo.

Describimos las variables utilizando frecuencias absolutas y relativas para las variables cualitativas, mientras que empleamos las medias y las desviaciones estándar para las variables cuantitativas. Para evaluar los factores asociados (perfil personal y profesional y hábitos diarios) con el síndrome de desgaste profesional y sus subdominios, construimos una tabla de contingencia y aplicamos la prueba de Chi-cuadrado para la independencia. En los casos en que los supuestos de los Chi-cuadrado no se cumplieron, aplicamos la prueba exacta de Fisher. Elaboramos un modelo de análisis bivariado para predecir nuestros resultados (presencia de agotamiento general y a través de sus 3 componentes) utilizando todas nuestras variables secundarias. De igual forma construimos el modelo invirtiendo las categorías de riesgo para evaluar posibles factores protectores en nuestra población de estudio. Todos los análisis se realizaron con un error Tipo I del 5\% y calculamos el intervalo de confianza (IC) asociado para cada parámetro relevante. Realizamos todos los cálculos con Stata 16 licencia del Hospital Militar Central de Bogotá. La investigación se llevó a cabo después del análisis y aprobación por parte del Comité de Ética de la Sociedad Colombiana de Cirugía Plástica, Estética y Reconstructiva.

\section{Resultados}

Regisgtramos 623 participantes de los cuales 132 participaron en la encuesta la cual fue una búsqueda activa en su mayoria. No se alcanzó el número total de participantes debido a la dificultad de acercarse a algunos de ellos o por la no devolución de los cuestionarios.

Del total de 132 profesionales encuestados, 100 fueron especialistas y 32 residentes de diferentes años de 
Tabla I. Variables sociodemográficas de especialista en Cirugía Plástica

\begin{tabular}{|c|c|c|c|}
\hline $\begin{array}{c}\text { Variable } \\
\mathrm{n}=100\end{array}$ & Categoria & $\mathbf{n}$ & $\%$ \\
\hline \multirow{2}{*}{ GÉNERO } & Hombre & 66 & 66 \\
\hline & Mujer & 34 & 34 \\
\hline EDAD (Media/ Ds) & & $47.6(10)$ & \\
\hline \multirow{4}{*}{ ESTADO CIVIL } & Casado & 66 & 66 \\
\hline & Soltero & 20 & 20 \\
\hline & Divorciado & 7 & 7 \\
\hline & Unión libre & 7 & 7 \\
\hline \multirow{5}{*}{ SUBESPECIALIDAD* } & Estetica & 90 & 90 \\
\hline & Mano & 21 & 21 \\
\hline & Microcirugía & 7 & 7 \\
\hline & Oncología & 17 & 17 \\
\hline & Quemados & 19 & 19 \\
\hline \multirow{2}{*}{ DOCENCIA } & SI & 36 & 36 \\
\hline & NO & 64 & 64 \\
\hline \multirow{2}{*}{ TABAQUISMO } & SI & 8 & 8 \\
\hline & NO & 92 & 92 \\
\hline \multirow{2}{*}{$\begin{array}{c}\text { CONSUMO DE } \\
\text { ALCOHOL SEMANAL }\end{array}$} & SI & 18 & 18 \\
\hline & $\mathrm{NO}$ & 82 & 82 \\
\hline \multirow{2}{*}{$\begin{array}{l}\text { ENFERMEDAD } \\
\text { INCAPACITANTE }\end{array}$} & SI & 3 & 3 \\
\hline & NO & 97 & 97 \\
\hline \multirow{2}{*}{ DEPRESIÓN MAYOR } & SI & 2 & 2 \\
\hline & NO & 98 & 98 \\
\hline \multirow{2}{*}{$\begin{array}{l}\text { HORAS TRABAJO } \\
\text { SEMANAL }\end{array}$} & $<48$ horas & 64 & 64 \\
\hline & $>48$ horas & 36 & 36 \\
\hline \multirow{2}{*}{$\begin{array}{l}\text { AÑOS ANTIGÜEDAD } \\
\text { LABORAL }\end{array}$} & $<15$ años & 59 & 59 \\
\hline & $>15$ años & 41 & 41 \\
\hline \multirow{3}{*}{$\begin{array}{c}\text { PERSONAS QUE } \\
\text { DEPENDEN } \\
\text { ECONÓMICAMENTE }\end{array}$} & Ninguno & 21 & 21 \\
\hline & $\begin{array}{l}\text { Entre } 1 \text { y } 5 \\
\text { personas }\end{array}$ & 69 & 69 \\
\hline & $>6$ personas & 10 & 10 \\
\hline \multirow{3}{*}{$\begin{array}{l}\text { NÚMERO DE } \\
\text { TRABAJOS }\end{array}$} & 1 & 39 & 39 \\
\hline & 2 a 3 & 50 & 50 \\
\hline & $4 \mathrm{Aa} 5$ & 11 & 11 \\
\hline \multirow{2}{*}{$\begin{array}{l}\text { NÚMERO DE HORAS } \\
\text { DE SUEÑO }\end{array}$} & $<7$ horas & 77 & 77 \\
\hline & $>7$ horas & 53 & 53 \\
\hline \multirow{2}{*}{$\begin{array}{l}\text { ACTIVIDAD DE } \\
\text { ESPARCIMIENTO }\end{array}$} & Actividad física & 33 & 33 \\
\hline & $\begin{array}{l}\text { Actividad } \\
\text { cultural }\end{array}$ & 53 & 53 \\
\hline \multirow{3}{*}{$\begin{array}{c}\text { HORAS /SEM } \\
\text { ACTIVIDAD FÍSICA }\end{array}$} & Ninguna & 24 & 24 \\
\hline & Entre 1 y 5 & 46 & 46 \\
\hline & $>5$ Horas & 30 & 30 \\
\hline \multirow{3}{*}{$\begin{array}{l}\text { TURNOS } \\
\text { NOCTURNOS } \\
\text { SEMANA } \\
\end{array}$} & Ninguno & 82 & 82 \\
\hline & $<2$ turnos & 15 & 15 \\
\hline & $>2$ turnos & 3 & 3 \\
\hline \multirow{3}{*}{ NUMERO DE HIJOS } & Ninguno & 35 & 35 \\
\hline & $<2$ Hijos & 50 & 50 \\
\hline & $>2$ Hijos & 15 & 15 \\
\hline
\end{tabular}

*Algunos especialistas tienen más de 1 subespecialidad especialización. Las Tablas I y II muestran las características descriptivas de la muestra de estudio.

En relación con el perfil personal de los especialistas, la edad promedio de los 100 que respondieron fue de 47.6 (DS 10), hombres el 66\%, casados el 66\%, no tienen hijos el 35\% y menos de 2 hijos el 50\%. El 86\% realizan alguna actividad de esparcimiento, siendo las actividades culturales las más frecuentes. Con respecto al perfil profesional, el $36 \%$ se dedica a la docencia, la mayoría trabajan menos de 48 horas semanales (64\%), no realizan turnos nocturnos (82\%) y el $90 \%$ practican la Cirugía Estética, seguida de Cirugía de Mano (21\%) y en menor porcentaje Microcirugía (7\%) (Tabla I).

En relación con el perfil personal de los residentes, la edad promedio fue de 30.8 años (DS 4.8); de los 32 residentes encuestados 14 fueron hombres (43.8\%) y 18 mujeres (56.2\%); solteros 25 (78.2\%), no tienen hijos $28(87.5 \%)$. El 100\% realizan alguna actividad de esparcimiento, siendo la actividad física las más frecuente $(71.9 \%)$. Con respecto al perfil profesional, 31 (96.8\%) realizan menos de 2 turnos nocturnos o ningún turno por semana.

Tabla II. Variables sociodemográficas de residentes de Cirugía Plástica

\begin{tabular}{|c|c|c|c|}
\hline $\begin{array}{c}\text { Variable } \\
n=32\end{array}$ & Categoria & $\mathbf{n}$ & $\%$ \\
\hline \multirow{2}{*}{ GÉNERO } & Hombre & 14 & 43.8 \\
\hline & Mujer & 18 & 56.2 \\
\hline EDAD (Media/ Ds) & & $30.8(4.8)$ & \\
\hline \multirow{4}{*}{ ESTADO CIVIL } & Casado & 6 & 18.7 \\
\hline & Soltero & 25 & 78.2 \\
\hline & Divorciado & 0 & 0 \\
\hline & Unión libre & 1 & 3.1 \\
\hline \multirow{2}{*}{ TABAQUISMO } & SI & 5 & 15.6 \\
\hline & $\mathrm{NO}$ & 7 & 84.4 \\
\hline \multirow{2}{*}{$\begin{array}{c}\text { CONSUMO DE } \\
\text { ALCOHOL SEMANAL }\end{array}$} & SI & 9 & 28.1 \\
\hline & $\mathrm{NO}$ & 23 & 71.9 \\
\hline \multirow{2}{*}{$\begin{array}{l}\text { ENFERMEDAD } \\
\text { INCAPACITANTE }\end{array}$} & $\mathrm{SI}$ & 0 & 0 \\
\hline & $\mathrm{NO}$ & 32 & 100 \\
\hline \multirow{2}{*}{ DEPRESIÓN MAYOR } & $\mathrm{SI}$ & 0 & 0 \\
\hline & $\mathrm{NO}$ & 32 & 100 \\
\hline \multirow{2}{*}{$\begin{array}{l}\text { ACTIVIDAD DE } \\
\text { ESPARCIMIENTO }\end{array}$} & $\begin{array}{c}\text { Actividad } \\
\text { física }\end{array}$ & 23 & 71.9 \\
\hline & $\begin{array}{c}\text { Actividad } \\
\text { cultural }\end{array}$ & 9 & 28.1 \\
\hline \multirow{3}{*}{$\begin{array}{c}\text { HORAS /SEM } \\
\text { ACTIVIDAD FÍSICA }\end{array}$} & Ninguna & 9 & 28.1 \\
\hline & Entre 1 y 5 & 17 & 53.1 \\
\hline & $>5$ Horas & 6 & 18.8 \\
\hline \multirow{3}{*}{$\begin{array}{c}\text { TURNOS NOCTURNOS } \\
\text { SEMANA }\end{array}$} & Ninguno & 11 & 34.3 \\
\hline & $<2$ turnos & 20 & 62.5 \\
\hline & $>2$ turnos & 1 & 3.2 \\
\hline \multirow{3}{*}{ NÚMERO DE HIJOS } & Ninguno & 28 & 87.5 \\
\hline & $<2$ Hijos & 4 & 12.5 \\
\hline & $>2$ Hijos & 0 & 0 \\
\hline
\end{tabular}


Tabla III. Análisis bi-variado del síndrome de desgaste profesional en especialistas de Cirugía Plástica en Colombia

\begin{tabular}{|c|c|c|c|c|c|c|c|}
\hline Variable & Categoria & $\begin{array}{c}\text { Alto } \\
\text { agotamiento } \\
\text { emocional } \\
\mathbf{n}=98\end{array}$ & $\mathbf{P}^{*}$ & $\begin{array}{c}\text { Alta } \\
\text { despersonalización } \\
\mathbf{n}=98\end{array}$ & $\mathbf{P} *$ & $\begin{array}{c}\text { Baja realización } \\
\text { personal } \\
n=98\end{array}$ & $\mathbf{P}^{*}$ \\
\hline \multirow{2}{*}{ GÉNERO } & Hombre & 10 & 0.2 & 2 & 0.3 & 7 & 0.5 \\
\hline & Mujer & 8 & & 3 & & 3 & \\
\hline \multirow{2}{*}{ ESTADO CIVIL } & Casado & 11 & 0.9 & 4 & 1.0 & 5 & 0.1 \\
\hline & Soltero & 5 & & 1 & & 3 & \\
\hline \multirow{2}{*}{ HORAS DE SUEÑO } & 7 o menos & 16 & 0.3 & 5 & 0.2 & 10 & 0.3 \\
\hline & 8 o más & 2 & & 0 & & 0 & \\
\hline \multirow{2}{*}{$\begin{array}{c}\text { HORAS DE TRABAJO } \\
\text { SEMANAL }\end{array}$} & 48 o menos & 8 & & 2 & & 1 & \\
\hline & Mayor a 48 & 10 & 0.06 & 3 & 0.2 & 9 & 0.6 \\
\hline \multirow{2}{*}{$\begin{array}{l}\text { ACTIVIDAD } \\
\text { ESPARCIMIENTO }\end{array}$} & Actividad fisica & 8 & 0.3 & 2 & 0.6 & 1 & \\
\hline & $\begin{array}{l}\text { Actividad } \\
\text { cultural }\end{array}$ & 8 & & 3 & & 8 & 0.07 \\
\hline \multirow{2}{*}{ TURNOS SEMANALES } & Ninguno & 5 & & 0 & & 3 & \\
\hline & Más de 2 & 12 & 0.05 & 5 & 0.1 & 7 & 0.2 \\
\hline \multirow{2}{*}{ NÚMERO DE HIJOS } & Ninguno & 6 & & 0 & & 5 & \\
\hline & Mas de 2 & 12 & 0.2 & 5 & 0.2 & 5 & 0.9 \\
\hline
\end{tabular}

*p valor obtenido a través de la prueba de Fisher

Tabla IV. Análisis bi-variado de bajo riesgo de síndrome de desgaste profesional en especialistas

\begin{tabular}{|c|c|c|c|c|c|c|c|}
\hline Variable & Categoria & $\begin{array}{c}\text { Bajo } \\
\text { agotamiento } \\
\text { emocional } \\
\mathbf{n}=98\end{array}$ & $\mathbf{P}^{*}$ & $\begin{array}{c}\text { Baja } \\
\text { despersonalización } \\
\mathbf{n}=98\end{array}$ & $\mathbf{P}^{*}$ & $\begin{array}{c}\text { Alta } \\
\text { realización } \\
\text { personal } \\
\mathrm{n}=98\end{array}$ & $\mathbf{P} *$ \\
\hline \multirow{2}{*}{ GÉNERO } & Hombre & 45 & 0.4 & 60 & 0.2 & 54 & 0.5 \\
\hline & Mujer & 18 & & 26 & & 26 & \\
\hline \multirow{2}{*}{ ESTADO CIVIL } & Casado & 47 & 0.04 & 4 & 0.6 & 53 & 0.8 \\
\hline & Soltero & 8 & & 1 & & 16 & \\
\hline \multirow{2}{*}{ HORAS DE SUEÑO } & 7 o menos & 49 & 0.3 & 5 & 0.2 & 10 & 0.3 \\
\hline & 8 o más & 15 & & 0 & & 0 & \\
\hline \multirow{2}{*}{$\begin{array}{l}\text { HORAS DE TRABAJO } \\
\text { SEMANAL }\end{array}$} & 48 o menos & 43 & 0.1 & 2 & & 52 & \\
\hline & Mayor a 48 & 20 & & 3 & 0.2 & 27 & 0.2 \\
\hline \multirow{2}{*}{$\begin{array}{l}\text { AÑOS ANTIGÜEDAD } \\
\text { LABORAL }\end{array}$} & $<15$ años & 30 & & 45 & 0.3 & 41 & 0.07 \\
\hline & $>15$ años & 32 & 0.02 & 39 & & 28 & \\
\hline \multirow{2}{*}{$\begin{array}{l}\text { NÚMERO DE } \\
\text { TRABAJOS }\end{array}$} & $<3$ trabajos & 61 & 0.03 & 80 & 0.1 & 74 & 0.2 \\
\hline & $>3$ trabajos & 9 & & 6 & & 6 & \\
\hline \multirow{2}{*}{$\begin{array}{l}\text { ACTIVIDAD DE } \\
\text { ESPARCIMIENTO }\end{array}$} & $\begin{array}{l}\text { Actividad } \\
\text { física }\end{array}$ & 17 & & 29 & & 31 & \\
\hline & $\begin{array}{l}\text { Actividad } \\
\text { cultural }\end{array}$ & 38 & 0.03 & 45 & 0.7 & 39 & 0.02 \\
\hline \multirow{3}{*}{$\begin{array}{c}\text { HORAS /SEM } \\
\text { ACTIVIDAD FÍSICA }\end{array}$} & Ninguna & 12 & & 18 & & 20 & \\
\hline & Entre 1 y 5 & 28 & & 41 & 0.01 & 35 & 0.5 \\
\hline & $>5$ horas & 24 & 0.2 & 27 & & 25 & \\
\hline \multirow{2}{*}{$\begin{array}{c}\text { TURNOS } \\
\text { SEMANALES }\end{array}$} & Ninguno & 55 & 0.04 & 69 & 0.1 & 67 & 0.1 \\
\hline & Más de 2 & 9 & & 17 & & 13 & \\
\hline \multirow{3}{*}{ NÚMERO DE HIJOS } & Ninguno & 14 & & 25 & & 23 & \\
\hline & Mas de 2 & 50 & 0.02 & 15 & & 12 & \\
\hline & Menos de 2 & 38 & & 46 & 0.02 & 55 & 0.02 \\
\hline
\end{tabular}

*p valor obtenido a través de la prueba de Fisher 
Para determinar si los profesionales encuestados tenían o no síndrome de desgaste profesional eliminamos del análisis a 2 pacientes que presentaron criterios diagnósticos de depresión mayor, por lo tanto, realizamos el análisis de especialistas sobre una muestra de 98 pacientes. El resultado del análisis fue que solo 2 personas de la muestra cumplían con los criterios clasificatorios de síndrome de desgaste profesional. El análisis por componentes mostró que 18 profesionales tenían agotamiento emocional (18.3\%, IC 95\%: 25.7-32.3), 5 presentaban alta despersonalización (5.1\%, IC 95\%: 13.3-22) y 10 baja realización personal (10.2\%, IC 95\%: 38.7-40.2).

$\mathrm{Al}$ realizar el análisis bi-variado con la prueba de Fisher (Tabla III) observamos una relación significativa entre el alto agotamiento emocional y el realizar más de 2 turnos nocturnos a la semana $(\mathrm{p}=0.05)$.
Al realizar el análisis bi-variado con la prueba de Fisher para evaluar posibles factores protectores (Tabla IV) observamos una relación significativa entre el bajo agotamiento emocional y estar casado, antigüedad laboral mayor de 15 años, tener menos de 3 trabajos, realizar actividades culturales de esparcimiento, no hacer turnos nocturnos a la semana y tener de 2 a 3 hijos $(\mathrm{p}<0.05)$. Por otra parte, practicar entre 1 y 5 horas de actividad física a la semana y tener 2 o menos hijos presentaron relación significativa $(\mathrm{p}<0.05)$ con una baja despersonalización; de igual forma hubo relación significativa entre alta realización personal y realizar actividades de esparcimiento como actividades culturales y tener 2 o menos hijos.

Al evaluar la presencia del síndrome de desgaste profesional entre los residentes de Cirugía Plástica, ninguno cumplía con los criterios clasificatorios. El análisis

Tabla V. Análisis bi-variado descriptivo de riesgo de síndrome de desgaste profesional en residentes de Cirugía Plástica en Colombia

\begin{tabular}{|c|c|c|c|c|c|c|c|}
\hline Variable & Categoria & $\begin{array}{c}\text { Alto } \\
\text { agotamiento } \\
\text { emocional } \\
n=32\end{array}$ & $P^{*}$ & $\begin{array}{c}\text { Alta } \\
\text { despersonalización } \\
\mathbf{n}=\mathbf{3 2}\end{array}$ & $\mathbf{P *}$ & $\begin{array}{c}\text { Baja } \\
\text { realización } \\
\text { personal } \\
\mathbf{n}=32\end{array}$ & $\mathbf{P}^{*}$ \\
\hline \multirow{2}{*}{ GÉNERO } & Hombre & 8 & & 2 & & 11 & 0.1 \\
\hline & Mujer & 10 & 0.1 & 2 & 0.5 & 9 & \\
\hline \multirow{2}{*}{ ESTADO CIVIL } & Casado & 4 & & 0 & & 5 & \\
\hline & Soltero & 14 & 1.0 & 3 & 0.1 & 15 & 0.1 \\
\hline \multirow{3}{*}{$\begin{array}{c}\text { HORAS /SEM } \\
\text { ACTIVIDAD FÍSICA }\end{array}$} & Ninguna & 9 & 0.3 & 2 & & 2 & \\
\hline & Entre 1 y 5 & 6 & & 2 & 0.8 & 4 & 0.5 \\
\hline & $>5$ horas & 3 & & 0 & & 1 & \\
\hline \multirow{2}{*}{$\begin{array}{c}\text { TURNOS } \\
\text { SEMANALES }\end{array}$} & Ninguno & 6 & & 1 & & 3 & \\
\hline & Más de 2 & 12 & 1.0 & 3 & 0.1 & 7 & 0.2 \\
\hline \multirow{3}{*}{ NÚMERO DE HIJOS } & Ninguno & 17 & 0.4 & 4 & 0.1 & 7 & 1.0 \\
\hline & Más de 2 & 1 & & 0 & & 0 & \\
\hline & Menos de 2 & 2 & & 3 & & 2 & \\
\hline
\end{tabular}

*p valor obtenido a través de la prueba de Fisher

Tabla VI. Análisis bi-variado descriptivo bajo riesgo de síndrome de desgaste profesional en Residentes de Cirugía Plástica en Colombia

\begin{tabular}{|c|c|c|c|c|c|c|c|}
\hline Variable & Categoria & $\begin{array}{c}\text { Bajo } \\
\text { agotamiento } \\
\text { emocional } \\
\mathbf{n}=\mathbf{3 2}\end{array}$ & $\mathbf{P} *$ & $\begin{array}{c}\text { Bajo } \\
\text { despersonalización } \\
\mathbf{n}=\mathbf{3 2}\end{array}$ & $\mathbf{P}^{*}$ & $\begin{array}{c}\text { Alta } \\
\text { realización } \\
\text { personal } \\
\mathbf{n}=\mathbf{3 2}\end{array}$ & $P^{*}$ \\
\hline \multirow{2}{*}{ GÉNERO } & Hombre & 4 & 0.1 & 12 & 0.5 & 4 & 0.6 \\
\hline & Mujer & 3 & & 9 & & 3 & \\
\hline \multirow{2}{*}{ ESTADO CIVIL } & Casado & 4 & & 3 & & 0 & \\
\hline & Soltero & 20 & 0.4 & 7 & 0.1 & 7 & 0.4 \\
\hline \multirow{3}{*}{$\begin{array}{c}\text { HORAS /SEM } \\
\text { ACTIVIDAD FÍSICA }\end{array}$} & Ninguna & 1 & & 9 & 0.6 & 11 & 0.4 \\
\hline & Entre 1 y 5 & 6 & 0.05 & 7 & & 6 & \\
\hline & $>5$ horas & 4 & & 5 & & 3 & \\
\hline \multirow{2}{*}{$\begin{array}{c}\text { TURNOS } \\
\text { SEMANALES }\end{array}$} & Ninguno & 10 & & 7 & & 6 & \\
\hline & Más de 2 & 15 & 1.0 & 14 & 0.1 & 14 & 0.3 \\
\hline \multirow{3}{*}{ NÚMERO DE HIJOS } & Ninguno & 23 & 0.03 & 17 & 0.06 & 16 & 0.4 \\
\hline & Más de 2 & 1 & & 2 & & 2 & \\
\hline & Menos de 2 & 3 & & 4 & & 4 & \\
\hline
\end{tabular}

*p valor obtenido a través de la prueba de Fisher 
de los diversos componentes del síndrome mostró las siguientes frecuencias entre los residentes: 18 con alto agotamiento emocional (56.2\%, IC 95\%: 30.3-44.3), 4 con alta despersonalización (12.5\%, IC 95\%: 28.3-42) y 7 con baja realización personal $(21.8 \%$, IC $95 \%$ : $30.8-$ 44.8) (Tabla V).

Al realizar el análisis bi-variado con la prueba de Fisher para evaluar posibles factores protectores (Tabla VI) observamos una relación significativa entre el bajo agotamiento emocional y no tener hijos y realizar entre 1 y 5 horas de actividad física semanal $(\mathrm{p}<0.05)$.

\section{Discusión}

El síndrome de desgaste profesional en el personal de la salud es una entidad que se ha empezado a estudiar de forma reciente. Múltiples publicaciones pretenden exaltar esta patología para empezar a generar estrategias no solo de manejo, sino también de prevención, con el objetivo de mejorar las condiciones laborales y evitar desenlaces que en el peor de los escenarios podrian involucrar a los pacientes. En Cirugía Plástica existe poca literatura respecto al tema, de hecho no hay, hasta donde hemos podido comprobar, un estudio enfocado a la especialidad o que incluya a residentes en formación.

Nuestra revisión del tema nos ha llevado a encontrar publicaciones nacionales e internacionales acerca de este fenómeno laboral en general, mostrando resultados que oscilan en las diferentes poblaciones sin dar un resultado claro de su prevalencia. Otra de las situaciones que desestiman las cifras publicadas es que se considera que el MBI-HSS, a pesar de ser un instrumento de medición, no se comporta como criterio diagnóstico oficial y algunos de sus componentes no difieren la etiología causante de los síntomas del paciente teniendo en cuenta que algunos pueden presentar problemas a nivel laboral por situaciones emocionales ocurridas en ambientes ajenos a éste. Sin embargo, es una herramienta útil y práctica que refleja la situación de muchos profesionales del sector salud y ha permitido una estandarización y aplicabildad del estudio, motivo por el cual está avalada internacionalmente y fue la razón para escogerla en nuestro estudio. ${ }^{(15-17)}$

El síndrome de desgaste profesional ha ganado mayor popularidad mediante el aumento de los estudios publicados en los últimos años, desde su primera publicación en el año 1978. De esta forma, se ha logrado que diferentes instituciones prestadoras de servicios de salud identifiquen esta problemática y desarrollen estrategias de prevención y manejo en su personal, enfocándonos no solo a tratar la enfermedad, sino a su prevención primaria.
En el 2014, Hansen y col. publicaron los resultados de una encuesta aplicada a 708 cirujanos plásticos de Estados Unidos, evidenciando que alrededor del 25\% de los mismos presentaban puntuaciones elevadas e identificaron como principales factores de riesgo la edad entre 40 y 50 años, problemas de salud en el cirujano, disponibilidad de llamada para servicios de urgencia y práctica principalmente en Cirugía Reconstructiva. ${ }^{(16)}$ Rawlani y col., un año después, publican los resultados de 1691 encuestados de entre 5942 invitados entre cirujanos plásticos de la Sociedad Americana de Cirugía Plástica, mostrando una tasa de afectación superior al $29.7 \%$ y señalando como principales factores de riesgo tener una subespecialidad, el número de horas trabajadas a la semana y los ingresos anuales. ${ }^{(17)}$ MedScape, en su reporte anual sobre burnout y suicidio en médicos en los Estados Unidos del año 2020, no solo evalúa la tasa de éste síndrome, sino que lo divide por especialidades médicas, encontrando las tasas más altas en Urología y Neurología con un 54\% y un 50\% respectivamente y en cirujanos plásticos con un 37\%, diferencia que atribuye a las situaciones de estrés que enfrentan cada una de estas especialidades. ${ }^{(18)}$

En Colombia en 2003, en la ciudad de Medellín, se realizó una muestra constituida por 60 participantes de un hospital entre los 20 y 40 años de edad evaluando los siguientes aspectos: la versión corta del inventario de Maslach sobre fatiga laboral, el índice de percepción del estrés laboral, el inventario sobre habilidades de enfrentamiento al estrés y una escala de apoyo social, donde el porcentaje de síndrome de desgaste profesional resultó bajo para esta población; sin embargo, un estudio publicado en 2017 por Domínguez y col. en un grupo de especialistas y residentes de Cirugía General en Colombia muestra una prevalencia del $33 \%$ en esta especialidad quirúrgica e insiste en que estas cifras no solo se presentan durante la residencia, sino que se mantienen incluso en los especialistas graduados, evidenciando la cronicidad de ésta entidad. ${ }^{(19)}$

En nuestro estudio, aplicamos el instrumento MBI durante un evento nacional a los especialistas y residentes de Cirugía Plástica que aceptaron participar de manera voluntaria, siendo esta, hasta donde sabemos, la primera vez que se incluye a un grupo de residentes de la especialidad para aplicar esta encuesta. Dentro de los resultados encontrados evidenciamos una tasa de desgaste profesional menor comparada con los demás estudios realizados $(2 \%)$, respecto a otro estudio de referencia en los Estados Unidos (29.7\%) hecho por Rawlani y col. ${ }^{(17)}$ y otro estudio en el mismo país realizado por Hansen con un $25 \%$ reportado, ${ }^{(16)}$ en el que no se estudia su incidencia entre los estudiantes de la especialidad. 
Para hablar del síndrome de desgaste profesional se incluyeron aquellas personas que cumplieron con un alto puntaje en agotamiento emocional y despersonalización y bajo en realización personal. Sin embargo, se considera internacionalmente personas en riesgo de desarrollarlo a aquellas que hayan cumplido con al menos 1 de los 3 ítems. En nuestro estudio, en el grupo de especialistas, hasta el $18 \%$ obtuvo puntuaciones altas en agotamiento emocional, el $5 \%$ en despersonalización y el $10 \%$ una puntuación baja en realización personal. En el grupo de residentes obtuvimos un mayor porcentaje, encontrando un $56 \%$ con alta puntuación en agotamiento emocional, un $12 \%$ alto en despersonalización y un $21 \%$ con baja puntuación en realización personal, siendo este último el grupo con mayor riesgo de desarrollar el síndrome, al igual que encontramos en los demás estudios realizados en Colombia sobre residentes de Cirugía General y de Ginecología.

En el grupo de especialistas, encontramos que aquellos que estaban casados, con un menor número de empleos y que realizaban actividades de esparcimiento, obtuvieron una mejor puntuación en la encuesta, hallazgo similar a los demás estudios mencionados. ${ }^{(17)} \mathrm{A}$ esto se sumó una mayor antigüedad laboral, la ausencia de turnos nocturnos y el tener hijos.

Por otra parte, en el grupo de residentes, aquellos que no tenían hijos, al igual que los que realizaban actividad física durante la semana, presentaron mejores puntuaciones en la encuesta.

Otro estudio publicado por Serenari y col. ${ }^{(20)}$ hace una comparación entre 190 residentes de especialidades quirúrgicas frente a no quirúrgicas, encontrando una prevalencia de 56.frente a $73 \%$ de esta entidad, siendo más frecuente en las especialiades no quirúrgicas.

Si bien la frecuencia del sindrome de desgaste profesional en nuestro estudio fue baja, logramos identificar aquellos aspectos sociodemográficos que pudieran influir en las personas con mejores puntuaciones. Esto con el fin de no solo centrarnos en los posibles factores de riesgo que puedan presentar los afectados, sino también en identificar aquellas características que podrían jugar un papel protector frente a esta entidad, como fueron el realizar actividad física y actividades de esparcimiento. Igualmente encontramos que un menor número de hijos y de trabajos se relacionó con menores puntuaciones.

Como limitación de nuestro estudio, señalar que contamos con un número reducido de participantes y que se basa en una encuesta puntual. Sin embargo, creemos que puede ser el inicio del estudio de esta patología tanto en especialistas como residentes de Cirugía Plástica no solo en Colombia, sino en Latinoamérica.

\section{Conclusiones}

El síndrome de burnout o de desgaste profesional en trabajadores de la salud es una entidad que, como se ha venido mencionando, requiere de mayor atención por parte de las instituciones. Afecta directamente al personal prestador de servicios de salud, a la calidad de atención a los pacientes, al ausentismo laboral y puede llevar a la pérdida de importantes sumas de dinero, incluyendo desenlaces fatales en los peores escenarios.

Estudios como el nuestro, en el que hacemos un estimado de la situación en Colombia tanto entre cirujanos plásticos especialistas como entre residentes en formación, permiten exponer aspectos psicosociales que podrían ser evaluados para identificar no solo factores de riesgo, sino también aquellos que juegan un rol protector para el desarrollo de esta patología.

En nuestro estudio encontramos una baja frecuencia del sindrome de desgaste profesional, siendo solo 2 de 98 especialistas los que cumplieron con criterios; igualmente, ninguno de los residentes encuestados presentó puntuaciones compatibles con esta entidad. Se asoció un mayor número de trabajos y de hijos con mayores puntuaciones en despersonalización y agotamiento emocional, al igual que un mayor número de horas semanales para actividad física y actividades de esparcimiento con menores puntuaciones.

Cabe resaltar que, en nuestro conocimiento, este es el primer estudio realizado entre cirujanos plásticos en Colombia y que no solamente evaluó especialistas, sino que se extendió tambien a médicos residentes.

De esta forma, creemos necesario no centrarnos únicamente en identificar y tratar a estos pacientes, sino también en proporcionar los medios para favorecer aquellos factores protectores que nos permitan la creación de estrategias de prevención y de esta forma, poder reducir la incidencia de esta patología.

Creemos también que este estudio puede ser replicable en diferentes paises y de esta forma se podría obtener una informacion más global que ayudaría a plantear medidas de prevención y mejoras enfocadas dentro de nuestra especialidad, que se traducirían en mejorar la relacion médico-paciente y a su vez en reducir la posibilidad de procesos jurídicos, que en ocasiones son tan frecuentes en nuestro medio.

\section{Dirección del autor}

Dr. John Vargas Rueda

Servicio de Cirugía Plástica

Hospital Militar Central de Colombia

Tv. 3C \#49-02, Comuna Chapinero

Bogotá, Colombia

Correo electrónico: johnjvr@gmail.com 


\section{Bibliografía}

1. Maslach C, Jackson S, Leiter M. Maslach Burnout Inventory. Consulting psychologists press, 1986, Vol. 21.

2. Romani M, Ashkar K. Burnout among physicians. Libyan $J$ Mediicine. 2014;9:1-6.

3. Hernandez P. Estudio Descriptivo del Síndrome de Burnout en Personal de Salud en el Hospital Militar De Bogotá. Acta Colomb Psicol. 2002;7:71-83.

4. Shanafelt T, Bradley K, Wipf J, Back A. Burnout and Self-Reported Patient Care in an Internal Medicine Residency Program. Ann Intern Med. 2002;136:358-367.

5. Vera G, Pivaral C, Vélez P, Palmer Y, Gómez-Vera A, Cabrera-Pivaral C, et al. Factores de Riesgo Organizacionales Asociados al Sindrome de Burnout en Medicos Anestesiólogos. Salud Ment. 2005;28:82-91.

6. Carlos J, Martínez A. Aspectos Epidemiológicos del Síndrome de Burnout en Personal Sanitario. Rev Esp Salud Publica. 1997;(3):293-303

7. Costa DO, Santos ISA, Ana II, Rodrigues T, Santos DA, Enaldo I. Burnout Syndrome and associated factors among medical students: a cross-sectional study. Clinics. 2012;67(6):573579.

8. Priebe S. Burnout Syndrome Among Physicians - The Role of Personality Dimensions and Coping Strategies. Psychiatr Dambina. 2011;23(4):389-395.

9. Correa Z, Muñoz I, Chaparro A. Síndrome de Burnout en docentes de dos universidades de Popayán, Colombia. Rev Salud Publica. 2010;12(4):589-598.

10. Quiroz R, Saco S. Factores Asociados al Sindrome Burnout en Medicos y Enfermeras del Hospital Nacional Sur Este de ESSALUD del Cusco. Rev Semestrla Fac Med Humana. 2003;12:1122.
11. Guevara CA, Henao DP, Herrera JA. Sindrome de Desgaste Profesional en Médicos Internos y Residentes. Hospital Universitario del Valle, Cali, 2002. Colomb Med. 2004;35:173-178.

12. Grau A, Suñer R, García M. Desgaste Profesional en el Personal Sanitario y su Relación con los Factores Personales y Ambientales. Gac Sanit. 2005;19(6):463-470.

13. Bernal JS, Ventura MF, Sevillano CP, Cabero SG, Cortés MD, Gutiérrez I, et al. Valores personales y profesionales en médicos de familia y su relación con el síndrome del burnout Introducción. An Psicol. 2006;22:45-51.

14. Córdoba, L., Tamayo, J.A., González, M.A., Martínez, M.I., Rosales, A., \& Barbato, G. Adaptation and validation of the Maslach Burnout Inventory - Human Services Survey in Cali, Colombia. Colombia Médica, 2011;42(3): 286-293.

15. Gil-monte PR, Peiró J. Perspectivas teóricas y modelos interpretativos para el estudio del síndrome de quemarse por el trabajo. An Psicol. 1999;15:261-268.

16. Streu R, Hansen $\mathbf{J}$, Abrahamse $P$, Alderman AK. Professional Burnout Among US Plastic Surgeons, Results of a National Survey. Ann Plast Surg. 2014;72(3):346-350.

17. Rawlani V. Burnout Phenomenon in US Plastic Surgeons: Risk Factors and Impact on QUality of Life. Plast Reconstr Surg. 2015;135(2):619-626

18. MedScape. Medscape National Physician Burnout \& Suicide Report 2020: The Generational Divide. Enero, 2020.

19. Dominguez L, Sanabria A, Ramirez A, Vargas F, Pacheco M, Jimenez G. Desgaste profesional en residentes colombianos de cirugía: resultados de un estudio nacional. Rev Colomb Cir. 2017;32:121-127.

20. Serenari M, Cucchetti A, Russo PM, Fallani G, Mattarozzi K, Pinna AD, Colonnello V, Poggioli G, Cescon M. Burnout and psychological distress between surgical and non-surgical residents. Updates Surg. 2019;71(2):323-330

\section{Comentario al artículo "Frecuencia del síndrome de desgaste profesional en un grupo de especialis- tas en Cirugía Plástica y estudiantes del posgrado en Colombia"}

\section{Isabel CARAVANTES}

Cirujano Plástico, Clínica Dermoplástica S.C. Hospital Español de México, Investigación en ciencias médicas UNAM - IPN, Ciudad de México, México.

Vocal Fundación Docente FILACP 2020-2022

Interesante punto de partida el que presentan Vargas y col. en su estudio al analizar una problemática conocida en el ámbito médico pero con escasos datos de influencia en nuestra especialidad y entorno geográfico ibero-latinoamericano.

La definición de conceptos y metodología de la investigación es primordial para estructurar el tipo de estudio de investigación que realizaremos, el concepto de frecuencia y prevalencia expresa diferencias en cuanto al desarrollo del tipo de estudio de investigación y herramientas de medición para obtener resultados válidos.

Los estudios transversales a través de la obtención de datos en un momento temporal (índices estáticos) en un periodo fijo, muestran, a mi juicio, una inconveniencia en patologías como el burnout por la variabilidad de situaciones que se experimentan en cada momento. En el estudio realizado por Vargas y col. se hizo la recopilación de datos y la aplicación de un instrumento de medición MBI-HSS (Burnout de Maslach - Human Services Survey adaptada) además de un cuestionario sociodemográfico para evaluar factores asociados al síndrome de desgaste profesional en un momento especifico, a un grupo de estudio con diferencias en grado, responsabilidades, estando en un curso fuera de su ciudad de residencia y de trabajo, fuera de su lugar de acción, fuera de su entorno diario, con diferencia en cuanto a su estado de ánimo por estar conviviendo con amigos con respecto al momento de estar trabajando, además de otras variables que podrían afectar el resultado final. ${ }^{(1)}$

Los estudios de investigación que se determinan como estudios de prevalencia de periodo son aquellos en los que se hace la obtención de datos de los casos presentes en cualquier momento durante un periodo especifico, ${ }^{(2)}$ lo que podría ser el caso del estudio realizado por Vargas 
y col., lo que conlleva tener además otros métodos de medición de resultados.

Los diferentes estudios reportados en la literatura tienen una característica y es que los autores seleccionan o adaptan el instrumento de medición obtenido de entre la amplia gama disponible, además de las adaptaciones que hacen los autores, como las que realizaron al MBIHSS Vargas y col. Estas adaptaciones pueden ser con base a diferentes criterios que no siempre se explican en algunas publicaciones, por tanto, los resultados que se obtienen están sujetos a tener una gran variabilidad y no son concluyentes, de ahí que podamos considerar que un futuro estudio, como el que proponen Vargas y col., podría desarrollar una metodología de investigación más estructurada para obtener resultados que puedan ser válidos en un grupo profesional internacional y más amplio.

Considero relevante estandarizar y desarrollar sistemáticamente estos estudios para evaluar por especialidad $y$, posiblemente también por grupo especifico poblacional de estudio, y hacer una integración de resultados de forma global.

\section{Respuesta al comentario de la Dra. I. Garavantes}

\section{John VARGAS RUEDA}

Deseo agradecer a la Dra. Caravantes su interés por nuestro artículo. Esta patología es un tema muy publicitado pero desafortunadamente no existen artículos específicos para nuestra especialidad, por lo cual, encontrar una referencia adaptable para nuestro entorno y poderlo replicar era un reto académico interesante que decidimos afrontar.

La recolección de los datos era el punto clave; estudios previos que han utilizado métodos electrónicos no han tenido la mejor recepción y, desafortunadamente, la vinculación y disposición del tiempo eran limitadas. Fue por ese motivo que la reunión anual que hacemos en nuestro país era la opción mas plausible con el punto a favor de tener a los residentes para vincularlos al estudio. Estos congresos sirven para tener a los colegas con mejor disposición para la participación, estar en un ambiente lejano al ejercicio diario de sus actividades y con un ambiente jovial, disminuyendo el sesgo estadístico; sin embargo, esta opción fue una postura personal, ya que, hasta donde conocemos, no existe un artículo con alguna recomendación especifica
Animo a los autores a establecer el tipo de estudio de investigación a realizar a mayor escala, el planteamiento del problema y, sobre todo, a estructurar la metodología de la investigación a desarrollar, analizando las posibles variables que puedan afectar los resultados, hacer las mediciones específicas con base al tipo de estudio y, de esta forma, obtener resultados relevantes en nuestra especialidad y en diferentes entornos geográficos del ámbito ibero-latinoamericano en base a los cuales articular pautas de prevención y actuación frente al síndrome de desgaste profesional entre especialistas y residentes en formación.

\section{Bibliografia}

1. Fuentes Ferrer ME., del Prado González N. Desde el laboratorio a la clínica, medidas de frecuencia y de asociación en epidemiología clínica. An Pediatr Contin. 2013;11(6):346-349.

2. ArmigónPallás JM, Jiménez Villa J. Métodos de investigación clínica y epidemiológica. 3.a ed. Madrid: Elsevier S.A. 2000.

para realizar el test con la fortuna de tener las pruebas completas por los participantes.

Leyendo sus apreciaciones comparto muchas de ellas, en especial la referencia a la metodología, más aún teniendo en cuenta mi objetivo de realizar este mismo artículo de forma más amplia e integrarlo. Sin embargo, entiendo también que las conductas, los ritmos de trabajo, las condiciones sociales, económicas, académicas, culturales, políticas (sistemas de salud), etc. de cada país varían entre si, lo que hace que sea atractivo pensar en poder desarrollar un estudio a gran escala con un representante de cada país que lidere la recolección del test, analizar los datos, publicar en conjunto y aprender entre nosotros. De esta forma podríamos adaptar o corregir algunos manejos con el objetivo de mejorar nuestra atención diaria en nuestra especialidad, todo con el fin en común: el paciente.

De nuevo agradezco las recomendaciones y la invito a colaborar desde México para que podamos, con la colaboración de otros en los demás países, replicar la investigación en Iberoamérica. 